\title{
Funções de covariâncias sobre polinômios B-splines para modelagem do crescimento de bovinos de corte: revisão
}

\author{
[Covariance functions on B-splines polynomials for modeling the growth of beef cattle: review]
}

\section{"Revisão/Review"}

\section{Diego Helcias Cavalcante ${ }^{1 *}$, Amauri Felipe Evangelista ${ }^{2}$, José Elivalto Guimarães Campelo ${ }^{3}$, Danielle Maria Machado Ribeiro Azevêdo ${ }^{4}$, Severino Cavalcante Sousa Júnior ${ }^{3}$}

\author{
${ }^{1}$ Departamento de Medicina Veterinária, Universidade Federal do Piauí (UFPI), Bom Jesus-PI, Brasil. \\ ${ }^{2}$ Programa de Pós-Graduação em Zootecnia, Universidade Federal do Paraná (UFPR), Curitiba-PR, Brasil. \\ ${ }^{3}$ Programa de Pós-Graduação em Ciência Animal, Universidade Federal do Piauí (UFPI), Teresina-PI, Brasil. \\ ${ }^{4}$ Empresa Brasileira de Pesquisa Agropecuária (Embrapa Meio-Norte), Teresina-PI, Brasil. \\ *Autor para correspondência/Corresponding author: E-mail: diegohelcias@ hotmail.com
}

\section{Resumo}

O objetivo é abordar assuntos sobre polinômios B-splines utilizados em funções de covariância por meio de modelos de regressão aleatória com a finalidade de predizer o potencial genético em bovinos de corte em características de crescimento. Nos modelos de regressão aleatória é possível decompor a trajetória da curva de crescimento por meio das mudanças nas covariâncias dos efeitos aleatórios. O tipo e a ordem de ajuste dos polinômios utilizados para modelagem do crescimento podem influenciar os resultados das avaliações genéticas, portanto, é necessário avaliar diferentes modelos para se auferir o mais harmônico. Nesse contexto, o uso de polinômios segmentados do tipo B (Funções B-splines) tem sido proposto como alternativa aos polinômios de Legendre, no ajuste das funções de covariância. Atualmente, alguns autores concluíram que o uso de polinômios B-splines nos modelos de regressão aleatória apresentou vantagens em relação aos demais, porém, ainda não está claro alguns aspectos quanto à quantidade, ordem e posição dos nós para modelagem da curva.

Palavras chaves: avaliação genética; Bos indicus; parâmetros genéticos.

\begin{abstract}
The objective is to discuss subjects on B-splines polynomials used in covariance functions using random regression models to predict genetic potential in beef cattle on growth traits. In the random regression models, it is possible to decompose the growth curve trajectory by means of changes in random effects covariances. In random regression models, it is possible to decompose the trajectory of the growth curve by changing the covariance of random effects. The type and order of adjustment of the polynomials used for growth models may have an influence on the results of genetic evaluations, so it is necessary to evaluate different models to obtain the most harmonic. The use of type B segmented polynomials (B-splines functions) has been proposed as an alternative to Legendre polynomials in the adjustment of covariance functions. Currently, some authors concluded that the use of B-splines polynomials in random regression models presented advantages over the alternative, however, aspects related to the amount, order, and position of the nodes for the curve modeling are still unclear.
\end{abstract}

Keywords: genetic evaluation; Bos indicus; genetic parameters.

\section{Introdução}

O sucesso da bovinocultura de corte depende, em grande parte da constante melhoria na constituição gênica dos rebanhos. Animais com

maior potencial para produção de carne permitem incrementar a produção por meio de técnicas de manejo reprodutivo que visem o melhoramento 
genético do rebanho (Albuquerque et al., 2007). Para isto, devem-se selecionar os melhores animais para serem reprodutores e, dessa forma, permitir que os seus genes, responsáveis pelo incremento no potencial genético médio da população, sejam repassados às gerações futuras (Dias et al., 2006). A predição do potencial genético em bovino de corte pode ser feita com base na análise da sua curva de crescimento, e essa curva é gerada pela regressão do peso do animal em função da idade. Por meio de medições sucessivas do peso dos animais é possível construir uma trajetória média da curva de crescimento da população, utilizando modelos de regressão aleatória. Nos modelos de regressão aleatória é possível estimar parâmetros genéticos para todas as idades e etapas da vida do animal (Meyer, 1999).

Diferentes tipos de polinômios podem ser usados para ajustar as funções de covariância nos modelos de regressão aleatória, dentre eles, o mais utilizado para estimação de parâmetros genéticos em bovinos de corte é o polinômio ortogonal de Legendre (Meyer, 2005; Sousa Júnior et al., 2010; Boligon et al., 2012; Ferreira et al., 2015). O tipo e a ordem de ajuste dos polinômios utilizados para modelar as trajetórias médias de crescimento podem influenciar os resultados das avaliações genéticas, portanto, a utilização de modelos estatísticos e metodologias de avaliação genética inadequadas levam à diminuição da acurácia e podem reduzir o progresso genético (Meyer, 2003; Boligon et al., 2009; Baldi et al., 2010). No estudo da curva de crescimento, quando o período de tempo analisado é muito extenso e/ou o número de observações é reduzido, o modelo de regressão pode necessitar de polinômios de Legendre de alta ordem (Meyer, 1999; Nobre et al., 2003; Sakaguti et al., 2003; Boligon et al., 2010). Segundo Meyer (2005), polinômios de alta ordem apresentam maior exigência computacional e instabilidade numérica, colocam grande ênfase nas observações dos extremos da curva, levando a estimativas errôneas.

O uso de polinômios segmentados do tipo B (Funções B-splines) tem sido proposto como alternativa no ajuste das funções de covariância, por permitir unir vários segmentos de polinômios de baixo grau em posições específicas, para formar uma só curva contínua (Meyer, 2003). O uso de polinômios B-splines apresenta vantagens por melhor ajustar os extremos da curva e por sua convergência ser mais rápida (Boligon et al., 2012). A utilização dos polinômios B-splines tem despertado interesse pelos pesquisadores, por apresentar resultados satisfatórios na modelagem dos efeitos fixos e aleatórios que faz parte da equação da curva de crescimento (Baldi et al., 2010; Boligon et al., 2012).

O maior desafio para a utilização destas funções segmentadas é a identificação adequada do número de intervalos necessários e a posição dos nós na curva, ou seja, o comprimento de cada segmento. Novos estudos sobre aplicação de funções B-splines em modelos de regressão aleatória são necessários para uma melhor avaliação genética dos bovinos de corte (Meyer, 2005).

Sendo assim, objetivou-se abordar assuntos sobre polinômios B-splines utilizados em funções de covariância por meio de modelos de regressão aleatória com a finalidade de predizer o potencial genético em bovinos de corte.

\section{Revisão de Literatura}

\section{A bovinocultura de corte no Brasil}

O Brasil é o maior exportador e o segundo maior produtor de carne bovina do mundo (Figura 1), portanto, a pecuária de corte é um dos pilares do agronegócio brasileiro e apresenta grande destaque na economia nacional. Apesar da grande importância econômica para o País, a cadeia produtiva da carne ainda opera com baixa eficiência (BRASIL, 2014).

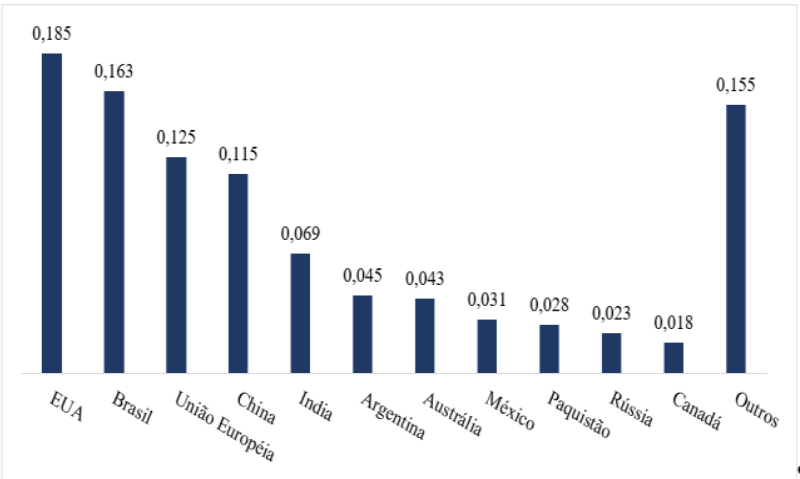

Figura 1. Produção mundial de carne bovina, em porcentagem (USDA, 2016).

O Brasil possui grande quantidade de terras destinada à pastagem, em torno de 170 milhões de hectares, com a produção de grãos se superando a cada ano (CONAB, 2015), porém, a produtividade relativa nos rebanhos de corte nacional ainda é baixa. Um dos motivos para a baixa produtividade é o uso de animais sem aptidão para produção de carne ou com potencial genético inapropriado para 
a região onde são criados (BRASIL, 2014). Essa ineficiência reflete em desperdício de insumos na produção animal.

Da população de bovinos criados no Brasil, cerca de $80 \%$ é composta por animais de espécie zebuína (Bos indicus), reconhecidos por serem animais rústicos, adaptados ao clima tropical, e por apresentarem maior resistência a endo e ectoparasitas, onde a maioria é criada a pasto (Malhado et al., 2005). Como consequência de baixo potencial genético dos animais e das pastagens, os índices produtivos destes animais são baixos e têm como um dos motivos a falta de programas de melhoramento genético abrangentes (Malhado et al., 2005).

Torna-se relevante a adoção de ferramentas tecnológicas, como a utilização de critérios de melhoramento genético animal, principalmente o uso de métodos de seleção e cruzamentos, bem como a união destes (Bueno et al., 2011), capazes de incrementar outros aspectos na bovinocultura de corte no Brasil, pois a maioria vem sendo voltada exclusivamente, à produção de carne.

Para a seleção dos melhores animais pelo desempenho produtivo em uma característica, devem-se levar em conta os parâmetros genéticos da mesma, além das correlações com as demais características de importância econômica (Paz et al., 1999). As características de crescimento corporal estão relacionadas, principalmente, à precocidade e à qualidade de carcaça, e têm grande destaque em programas de melhoramento por estarem ligadas diretamente ao ganho econômico para o produtor e atenderem às necessidades do mercado consumidor (Paz et al., 1999).

São características que podem ser mensuradas repetidas vezes no decorrer da vida do animal, e por isso são chamadas de medidas repetidas ou longitudinais. Dentre as características de interesse no progresso genético, o peso é uma medida longitudinal adotada para o monitoramento do crescimento de bovinos de corte, e pode ser obtida em larga escala a um custo baixo (Dias et al., 2006).

\section{Modelagem do crescimento animal}

Dados de crescimento mensurados em um mesmo indivíduo, do nascimento até a fase adulta, descrevem uma trajetória, chamada de curva de crescimento animal (Santoro et al., 2005). Dentre as medidas corporais utilizadas para a modelagem de crescimento, uma das mais comuns, considerada de fácil mensuração e que pode ser aferida em grandes populações por um custo relativamente baixo, é o peso do animal em determinadas idades (Albuquerque et al., 2007).

A regressão do peso em função da idade do animal, por meio de sucessivas pesagens ao longo do tempo, pode ser agrupada em parâmetros que são necessários para a análise genética, que servem de norte para a tomada de decisão na atividade (Santoro et al., 2005). O método de análise de dados longitudinais é de grande relevância para o melhoramento genético.

Nessas análises, diversos fatores devem ser levados em conta, como: raça (ou grupos genéticos), sexo, ano de nascimento, manejo (entre outros efeitos sistemáticos), como também, o potencial individual, atribuído ao valor genético aditivo de cada indivíduo (Freitas, 2005). Portanto, considerando-se que o fenótipo de um animal é a soma de efeitos fixos e aleatórios, a magnitude desses efeitos, bem como os parâmetros a ele atribuídos, também se modificam à medida que o animal fica mais velho (Freitas, 2005).

A análise da curva de crescimento permite decompor a variância fenotípica identificando variações decorrentes de fatores fixos e aleatórios, principalmente a variância genética aditiva, variância de ambiente e a variância residual, considerando as diferentes classes de idade, as quais caracterizam as fases do desenvolvimento corporal (Sousa Júnior et al., 2010).

Tendo em vista a padronização das análises genéticas, em programas de melhoramento são estabelecidas idades nos quais os pesos são ajustados. Essas idades são definidas como idadespadrão, que contemplam princípios biológicos como, por exemplo: peso ao nascimento, desmama, ano, sobreano e abate (Sakaguti et al., 2003). Entretanto, essas pesagens são difíceis de serem padronizadas durante a coleta, dificultando o processo de avaliação genética.

Para análise de regressão do peso do animal sobre sua idade, através de medidas repetidas no tempo, são indicados os modelos de regressão aleatória por propiciar a curva média de crescimento dos animais (Sousa Júnior et al., 2010). Uma das vantagens do uso de modelos de regressão aleatória é que permitem identificar as variações decorrentes de fatores fixos e aleatórios em qualquer idade do animal, sendo dispensável o uso de idades-padrão para análises genéticas (Resende et al., 2001). 


\section{Modelos de regressão aleatória e funções de covariância}

Os modelos de regressão aleatória e as funções de covariância podem expressar de maneira mais realista, os fenômenos associados a dados longitudinais, por exemplo, curvas de crescimento através do tempo ou medições repetidas (Resende et al., 2001).

Com a evolução das ferramentas computacionais, os modelos de regressão aleatória têm se tornado a metodologia padrão para análises de medidas longitudinais no melhoramento animal (Palharim et al., 2013). A utilização desses modelos permite o uso de forma eficiente de toda a informação disponível de cada animal, já que todas as medidas do animal e de seus parentes são utilizadas para a avaliação genética (Meyer, 2005), com isso, aumenta a acurácia das estimativas dos valores genéticos preditos.

Os modelos de regressão aleatória são adequados para explicar a variabilidade associada a medidas repetidas, quando se compara aos modelos lineares simples, pois permitem a obtenção de diferentes curvas de valores genéticos associadas aos diferentes indivíduos e considera as mudanças nas variâncias genéticas e residuais através do tempo (Resende et al., 2001). Estes modelos permitem a predição de valores genéticos de um indivíduo em diferentes idades, baseando-se em avaliações de apenas uma idade (Resende et al., 2001).

Isso ocorre devido às mudanças na média e variâncias da característica ao longo do tempo, geradas pelas funções de covariâncias. Estas funções, quando estimadas por regressão aleatória, são úteis para análise de dados de crescimento dos animais Nelore e aplicadas visando o progresso genético destes (Boligon et al., 2010; Santos et al., 2011; Palharim et al., 2013).

Ao se modelar a curva de variância como uma regressão em função de uma covariável contínua, os modelos de regressão aleatória são facilmente implementados em análises de modelos lineares mistos, considerando a influência de efeitos fixos e aleatórios (Meyer, 2005). Em avaliações genéticas da curva de crescimento, o ajuste de uma função contínua fixa é denominado de trajetória média de crescimento (Kirkpatrick et al., 1990). Ela representa a tendência de crescimento do peso corporal médio da população.

As curvas relativas ao efeito aleatório são, portanto, variações individuais dos animais que indicam o seu efeito genético e, de um modo geral, essas variações podem ser entendidas como desvios da produção dos animais em torno da curva fixa (Silva et al., 2011). Os modelos de regressão aleatória consideram diferentes estruturas de heterogeneidade de variância residual por meio da formação de classes de resíduo ao longo das idades de avaliação coletadas.

A importância de se considerar a variância residual heterogênea reside no fato de que se pode melhorar a partição da variância total, o que proporciona a estimação de parâmetros e valores genéticos mais acurados. Esse tipo de modelagem da variância residual pode aumentar o número de parâmetros a serem estimados no processo de maximização da função de verossimilhança (El Faro e Albuquerque, 2005).

O número elevado de parâmetros no modelo, a estrutura de (co)variância e o fato de as matrizes de incidência serem mais densas que as dos modelos convencionais podem dificultar a convergência na estimação dos componentes de variância, sendo entraves para a utilização dessa metodologia para avaliações genéticas em situações com grande número de dados (Meyer, 1999; Nobre et al., 2003; Sakaguti et al., 2003).

As funções de covariância são modeladas por polinômios, e de modo geral, do tipo ortogonal (Baldi et al., 2010). No entanto, polinômios alternativos como os segmentados, ou também conhecidos por funções Splines, podem ser utilizados sem modelos de regressão aleatória apresentando um melhor ajuste à curva (Baldi et al., 2010).

\section{Funções B-splines}

Funções Splines ou polinômios segmentados são curvas formadas por inúmeros segmentos de polinômios de menor grau, que se unem entre si por meio de posições determinadas, denominadas nós, formando assim uma curva contínua (Baldi et al., 2010). Tais nós (pontos de união) apontam o local em que ocorre a mudança de comportamento da curva, esse fenômeno é denominado de grau de polinômio ou inclinação (Paz et al., 1999).

Uma das extensões das funções Splines, são conhecidas por funções B-splines, onde a mesma é formada por segmentos de polinômios que compõem uma base de espaços Splines apresentando como vantagens a alta flexibilidade $\mathrm{e}$ rápida computação. As funções B-splines além de possuir as melhores propriedades numéricas, são indicadas na modelagem de efeitos aleatórios 
quando são analisados em modelos mistos para estimação de covariâncias.

Essas funções podem ser usadas de diversas maneiras, por meio de bases diferentes. Tholon et al. (2012) descreveram um modelo geral de um polinômio segmentado da seguinte forma:

$$
\begin{aligned}
Y=a_{0}+a_{1} X & +a_{2} x^{2}+b_{1} Z_{1}+b_{2} Z_{2}+b_{3} Z_{3} \\
& +b_{4} Z_{4}
\end{aligned}
$$

\section{Em que:}

$$
\begin{aligned}
& Z_{1}=\left(X-K_{1}\right)^{2}, \text { se } X>K_{1} ; \\
& Z_{2}=\left(X-K_{2}\right)^{2} \text {, se } X>K_{2} ; \\
& Z_{3}=\left(X-K_{3}\right)^{2}, \text { se } X>K_{3} ; \\
& Z_{4}=\left(X-K_{4}\right)^{2} \text {, se } X>K_{4} . \\
& Y=\text { é o peso, } X=\text { é a idade, } a_{0}=\text { é o }
\end{aligned}
$$
intercepto, $a_{1}$ e $a_{2}=$ são os parâmetros lineares e quadráticos, $b_{1}, b_{2}, b_{3}$ e $b_{4}=$ são os coeficientes de regressão dos polinômios e $\mathrm{K}_{1}, \mathrm{~K}_{2}, \mathrm{~K}_{3}$ e $\mathrm{K}_{4}=$ representam os nós.

O maior desafio na utilização destas funções é identificar a localização e a quantidade de nós para uma modelagem satisfatória da curva. O polinômio B-spline quadrático com quatro intervalos se ajustou adequadamente à trajetória média de crescimento de tourinhos Nelore, porém, quando avaliadas as trajetórias médias de crescimento de tourinhos mestiços Nelore e Canchim, o melhor ajuste foi obtido com uso de polinômios quadráticos do tipo B-splines com seis intervalos (Scalez et al., 2011).

Boligon et al. (2012), estudando o modelo mais adequado para descrever a estrutura de covariância dos dados de bovinos Nelore para os efeitos aleatórios, encontraram o polinômio Bspline quadrático, com três segmentos para efeito genético aditivo direto $\mathrm{e}$ efeito ambiental permanente animal e um segmento para efeito genético aditivo materno e efeito ambiental materno permanente. Este modelo obteve melhor ajuste aos dados, também, quando comparado com modelos que utilizaram polinômios de Legendre.

Baldi et al. (2010), encontrou resultados semelhantes a este estudo, porém, com bovinos da raça Canchim. O modelo de ajuste B-spline quadrático, com quatro nós ou três segmentos para efeito genético aditivo direto e efeito ambiental permanente animal e dois nós para efeito genético aditivo materno e efeito ambiental materno permanente, foi o mais adequado para descrever a estrutura de covariância dos dados.

O emprego de polinômios segmentados Bsplines nos estudos genéticos do crescimento de bovinos de corte busca esclarecer a ordem apropriada de ajuste das funções polinomiais para os diferentes efeitos aleatórios e a melhor estrutura de variância para modelar os resíduos.

\section{Considerações Finais}

O objetivo dos programas de melhoramento de bovinos de corte é alterar geneticamente uma população de indivíduos e as médias das características de interesse econômico, de modo que o lucro nos sistemas de produção aumente. Para isso, faz se o uso de modelos estatísticos nas avaliações genéticas, para identificar corretamente os valores genéticos dos animais candidatos à seleção.

$\mathrm{O}$ desafio é encontrar os modelos mais adequados, que são capazes de descrever satisfatoriamente os componentes de covariância dos efeitos aleatórios e, dessa forma, particionar corretamente a variação fenotípica total. O uso dos modelos de regressão aleatória ajustados por funções B-splines para estimar os componentes de variâncias e parâmetros genéticos, tem sido satisfatório pelos pesquisadores e traz benefícios, pois o mesmo é adequado para avaliação genética em características de crescimento, podendo ser recomendado para avaliação genética em bovinos de corte (Meyer, 2003; Boligon et al., 2009; Baldi et al., 2010; Scalez et al., 2011).

\section{Referências}

Albuquerque, L.G.; Mercadante, M.E.Z.; Eler, J.P. Aspectos da seleção de Bos indicus para produção de carne. Boletim de Indústria Animal, 64(4): 339-348, 2007.

Baldi, F.; Alencar, M.M.; Albuquerque, L.G. Random regression analyses using B-splines functions to model growth from birth to adult age in Canchim cattle. Journal of Animal Breeding and Genetics, 127(6): 433-441, 2010.

Boligon, A.A.; Silva, J.A.V.; Sesana, R.C.; Sesana, J.C.; Junqueira, J.B.; Albuquerque, L.G. Estimation of genetic parameters for body weights, scrotal circumference, and testicular volume measured at different ages in Nellore cattle. Journal of Animal Science, 88(4): 1215-1219, 2010.

Boligon, A. A.; Mercadante, M. E. Z.; Lôbo, R. B.; Baldi, F.; Albuquerque, L.G.D. Random regression analyses using $\mathrm{B}$-spline functions to model growth of Nellore cattle. Animal, 6(2): 212-220, 2012.

BRASIL. Ministério da Agricultura, Pecuária e Abastecimento, 2014. Plano mais pecuária. 
Disponível em: < http://www.agricultura.gov.br/assuntos/camar as-setoriais-tematicas/documentos/camarassetoriais/leite-e-derivados/anosanteriores/plano-mais-pecuaria.pdf/view >. Acesso em: 13 out. 2019.

Bueno, R.S.; Torres, R.A.; Ferraz, J.B.S.; Lopes, P.S.; Eler, J.P.; Silva, M.; Mattos, E.C. Inclusão da epistasia em modelo de avaliação genética de bovinos de corte compostos. Arquivo Brasileiro de Medicina Veterinária e Zootecnia, 63(4): 948-953, 2011.

CONAB. Indicadores da Agropecuária: Quadro de Suprimentos. Disponível em <http://www.conab.gov.br/conteudos.php?a= 1470\& $\mathrm{t}=2>$ Acesso em junho de 2016.

Dias, L.T.; Albuquerque, L.G.D.; Tonhati, H.; Teixeira, R. D. A. Estimação de parâmetros genéticos para peso do nascimento aos 550 dias de idade para animais da raça Tabapuã utilizando-se modelos de regressão aleatória. Revista Brasileira de Zootecnia, 35(5): 1915-1925, 2006.

El Faro, L.; Albuquerque, L.G. Predição de valores genéticos para a produção de leite no dia do controle e para produção acumulada até 305 dias. Revista Brasileira de Zootecnia, 34(2): 496-507, 2005.

Freitas, A.R. Curvas de crescimento na produção animal. Revista Brasileira de Zootecnia, 34(3): 786-795, 2005.

Kirkpatrick, M.; Lofsvold, D.; Bulmer, M. Analysis of the inheritance, selection and evolution of growth trajectories. Genetics, 124(4): 979-993, 1990.

Malhado, C.H.M.; Martins-Filho, R.; Lôbo, R.N.B.; Facó, O.; Azevedo, D.M.M.R.; Souza, J.C.; Oliveira, S.M.P. Tendências genéticas para características relacionadas à velocidade de crescimento em bovinos Nelore na região Nordeste do Brasil. Revista Brasileira de Zootecnia, 34(1): 60-65, 2005.

Meyer, K. Estimates of genetic and phenotypic covariance functions for post weaning growth and mature weight of beef cows. Journal of Animal Breeding and Genetics, 116(3): 181205, 1999.

Meyer, K. Estimates of genetic covariance functions for growth of Australian Angus cattle from random regression models fitting different orders of polynomials. Age (days), 100(300): 500, 2003.
Meyer, K. Random regression analyses using Bsplines to model growth of Australian Angus cattle. Genetics Selection Evolution, 37(5): 473-500, 2005.

Nobre, P.R.C.; Misztal, I.; Tsuruta, S.; Bertrand, J.K.L.; Silva, O.C.; Lopes P.S. Analyses of growth curves of Nelore cattle by multipletrait and random regression models. Journal Animal Science, 81(4): 918-926, 2003.

Palharim, D.A.; Araújo, C.V.; Bittencour, T.C.B.S.C.; Araújo, S.I.; Lôbo, R.B.; Bezerra, L.A.F. Estimativas de herdabilidade para o desempenho ponderal na raça Nelore no Mato Grosso. Scientific Electronic Archives, 3(1): 59-62, 2013.

Paz, C.C.P.; Albuquerque, L.G.; Fries, L.A. Efeitos ambientais sobre ganho de peso no período do nascimento ao desmame em bovinos da raça Nelore. Revista Brasileira de Zootecnia, 28(1): 55-64, 1999.

Resende, M.D.V.; Rezende, G.D.S.P.; Fernandes, J.S.C. Regressão aleatória e funções de covariância na análise de medidas repetidas. Revista de Matemática e Estatística, 19: 2140, 2001.

Sakaguti, E.S.; Silva, M.A.; Quaas, R.L.; Martins, E.N.; Lopes, P.S.; Silva, L.O.C. Avaliação do crescimento de bovinos jovens da raça Tabapuã, por meio de análises de funções de covariâncias. Revista Brasileira de Zootecnia, 32(4): 864-874, 2003.

Santoro, K.R.; Barbosa, S.B.P.; Santos, E.S.; Brasil, L.H.A. Herdabilidades de parâmetros de curvas de crescimento não-lineares em zebuínos, no Estado de Pernambuco. Revista Brasileira de Zootecnia, 34(6): 2280-2289, 2005.

Santos, G.C.J.; Lira, T.S.; Pereira L.S.; Lopes, F B.; Ferreira, J.L. Efeitos não genéticos sobre características produtivas em rebanhos Nelore criados na região norte do Brasil. Acta Veterinária Brasilica, 5(4): 385-392, 2011.

Scalez, D.C.B.; Fragomeni, B.O.; Passafaro, T.L.; Pereira, I.G.; Toral, F.L.B. Polynomials to model the growth of young bulls in performance tests. Animal, 8(3): 370-378, 2014.

Silva, F.L.; Alencar, M. M.; Freitas, A.R.; Packer, I.U.; Mourão, G.B. Curvas de crescimento em vacas de corte de diferentes tipos biológicos. Pesquisa Agropecuária Brasileira, 46(3): 262-271, 2011. 
Sousa Júnior, S.C.; Oliveira, S.M.P.D.; Albuquerque, L.G.D.; Boligon, A.A.; Martins Filho, R. Estimação de funções de covariância para características de crescimento da raça Tabapuã utilizando modelos de regressão aleatória. Revista Brasileira de Zootecnia, 39(5): 1037-1045, 2010.

Tholon, P.; Paiva, R.D.M.; Mendes, A.R.A.;

Barrozo, D. Utilização de funções lineares e não lineares para ajuste do crescimento de bovinos Santa Gertrudis, criados a pasto. Ars Veterinaria, 28(1): 234-239, 2012.

USDA. United State Department of Agriculture. Disponível em <http://www.fas.usda.gov/regions/brazil >. Acesso em 10 mar. 2018. 